\title{
Correction to: Site and Organ-Specific Culture-Dependent Endophytic Diversity of Crocus sativus L. (Saffron) in Kashmir Himalaya, India
}

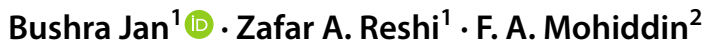

Published online: 26 August 2021

○) Springer Science+Business Media, LLC, part of Springer Nature 2021

\section{Correction to: Microbial Ecology \\ https://doi.org/10.1007/s00248-021-01817-5}

Due to typesetting mistake, the bottom part of Figure $7 \mathrm{e}$ was inaccurately trimmed. The original version has been corrected.

The original article can be found online at https://doi.org/10.1007/ s00248-021-01817-5

Bushra Jan

janbushra1@gmail.com

1 Department of Botany, University of Kashmir, Hazratbal,

Srinagar, Jammu and Kashmir 190006, India

2 Section of Plant Pathology, MRCFC, Khudwani

Sher-e-Kashmir University of Agricultural Sciences and Technology, Kashmir 190025, India 